\title{
BMJ Open Disrupted care during the COVID-19 state of emergency and productivity loss attributed to presenteeism in workers: a nationwide cross-sectional study
}

\author{
Tomohiro Ishimaru (D) , ${ }^{1}$ Kanami Tsuno, ${ }^{2}$ Ai Hori (D) , ${ }^{3}$ Makoto Okawara, ${ }^{1}$ \\ Yoshino Yasuda, ${ }^{1}$ Yoshihisa Fujino (D) , ${ }^{1}$ Takahiro Tabuchi (D) ${ }^{4}$
}

To cite: Ishimaru T, Tsuno $\mathrm{K}$, Hori A, et al. Disrupted care during the COVID-19 state of emergency and productivity loss attributed to presenteeism in workers: a nationwide crosssectional study. BMJ Open 2021;11:e050068. doi:10.1136/ bmjopen-2021-050068

- Prepublication history for this paper is available online. To view these files, please visit the journal online (http://dx.doi. org/10.1136/bmjopen-2021050068).

Received 09 February 2021 Accepted 24 November 2021

Check for updates

(c) Author(s) (or their employer(s)) 2021. Re-use permitted under CC BY-NC. No commercial re-use. See rights and permissions. Published by BMJ.

${ }^{1}$ Department of Environmental Epidemiology, Institute of Industrial Ecological Sciences, University of Occupational and Environmental Health, Japan, Kitakyushu, Japan

${ }^{2}$ School of Health Innovation, Kanagawa University of Human Services, Kawasaki, Japan ${ }^{3}$ Department of Global Public Health, University of Tsukuba, Tsukuba, Japan

${ }^{4}$ Cancer Control Center, Osaka International Cancer Institute, Osaka, Japan

Correspondence to

Dr Tomohiro Ishimaru;

ishimaru@med.uoeh-u.ac.jp

\section{ABSTRACT}

Objectives The COVID-19 pandemic has caused medical care delays and avoidance around the globe. However, little is known about the relationship between disrupted care and productivity loss attributed to presenteeism during the COVID-19 pandemic. We aimed to investigate whether disrupted care during the COVID-19 state of emergency was associated with health status and productivity loss.

Methods We used data from a nationwide, crosssectional, Internet-based, self-administered survey. We performed multiple logistic regression analysis on data from 14545 participants to investigate the associations among variables related to disrupted care, health status and the Work Functioning Impairment Scale, with a cut-off of 21 points.

Results Participants who experienced exacerbation of underlying disease (adjusted OR (aOR) 2.84; 95\% Cl 2.28 to 3.53 ) or any type of disrupted care were more likely to show low productivity at work. Experiencing disruptions in routine and non-routine clinical settings (aOR 4.64; $95 \% \mathrm{Cl} 3.64$ to 5.92 and a0R $6.29 ; 95 \% \mathrm{Cl} 4.74$ to 8.34 , respectively), and running out of drugs (aOR $6.13 ; 95 \% \mathrm{Cl}$ 4.60 to 8.18 ) were strongly associated with exacerbation of underlying disease.

Conclusions Workers who experienced disrupted care were much more likely to show productivity loss. Exacerbation of underlying disease is one possible pathway through which disrupted care could affect productivity loss attributed to presenteeism. Our study provides evidence of the importance of early diagnosis and continuous treatment of non-COVID-19 patients to enable them to remain healthy and continue to work during the pandemic.

\section{INTRODUCTION}

Presenteeism, the phenomenon of attending work despite being ill, is closely related to work performance. ${ }^{1}$ It is a global challenge for organisations because it affects workers' productivity and future sickness absence. ${ }^{2}$ According to Johns' theoretical framework, ${ }^{3}$ health status is the basis of presenteeism, and extrinsic pressures and intrinsic
Strengths and limitations of this study

- This study provides evidence of the importance of early diagnosis and continuous treatment of nonCOVID-19 patients to enable them to remain healthy and continue to work during the pandemic.

- This study revealed an occupational health problem emerging during the COVID-19 pandemic and drew on a large nationwide sample.

- The study used a cross-sectional design, so causal associations among disrupted care, health status and productivity loss could not be established.

- We did not specify which underlying disease was associated with disrupted care.

motivations strongly influence the choice of whether to go to work when experiencing ill health. Health status is associated with a variety of underlying diseases and conditions, including heart disease, depression, diabetes and low back pain. ${ }^{45}$ The strongest extrinsic drivers of presenteeism are strict sick leave policies, heavy workloads and staffing difficulties. ${ }^{6}$ In terms of intrinsic motivational paths, presenteeism is also more likely to occur with low job satisfaction and economic difficulty. ${ }^{6}$ The COVID-19 pandemic may affect presenteeism, including through the health status of workers. $^{7}$

The COVID-19 pandemic has caused medical care delays and avoidance around the globe..$^{8-12}$ Japan has the lowest morbidity and mortality associated with COVID-19 among all developed countries. A state of emergency was declared on 7 April 2020, for a specific region and then expanded to the whole country from 16 April to 25 May $2020 .{ }^{13}$ All citizens were asked to cancel non-essential appointments and stay at home during this period. In contrast to the situation in many other countries, the Japanese government did not implement a 'lockdown' (city blockade), but the non-compulsory 
state-of-emergency measures had a major impact on human movement. ${ }^{14}$ For example, a nationwide prescription database shows that the volume of otolaryngology prescriptions decreased by $55 \%$ and that the number of days of medication per prescription increased by $140 \%$, compared with the same period in $2019 .^{15}$

Reduced access to care during the state of emergency may have influenced workers' health status and productivity. A previous study has reported that the COVID-19 pandemic has had an indirect effect on excess deaths from chronic diseases because of temporary disruptions of care. ${ }^{16}$ On the basis of Johns' theoretical model, we hypothesised that disrupted care has a negative impact on workers' health status, resulting in lower productivity. ${ }^{3}$ Many findings on presenteeism behaviour during the COVID-19 pandemic have been reported. Implementing flexible, non-punitive paid leave and supportive measures as part of a comprehensive approach to preventing and reducing COVID-19 transmission among employees can have a positive impact on presenteeism behaviour. ${ }^{7}$ Conversely, major shifts in working practices, such as a change to working from home, and economic difficulties caused by the pandemic have been found to be strongly associated with presenteeism behaviour. ${ }^{717}$

We hypothesised that disrupted care during the state of emergency had a negative impact on workers' health status and presenteeism, resulting in productivity loss. For example, workers with back pain are often faced with productivity loss owing to their pain. ${ }^{18}$ However, if they exhaust their supply of painkillers, the pain may worsen and further reduce their productivity. Another example is that if depressed workers have been unable to see a doctor, they may continue to work as their condition worsens owing to a lack of medical advice about sick leave. However, little is known about the relationship between disrupted care and productivity loss attributed to presenteeism during the COVID-19 pandemic. Therefore, we aimed to investigate whether disrupted care during the COVID-19 state of emergency was associated with health status and productivity loss.

\section{METHODS}

\section{Study design and participants}

This study used data from the Japan COVID-19 and Society Internet Survey (JACSIS). This was a nationwide, crosssectional, Internet-based survey. Self-administered questionnaires were answered anonymously from 25 August to 30 September 2020. The population comprised panellists aged 15 to 79 years who were registered with an Internet research company (approximately 2.2 million people). Simple random sampling was used to select a survey population of 223389 people, who were invited via email to complete the survey. Participation was fully voluntary. After transitioning to the linked survey site, participants provided informed consent. We recruited participants in clusters by sex, age (in 5-year age bands) and prefecture (47 regions) to reflect nationally representative statistics. ${ }^{19}$ We stopped recruitment

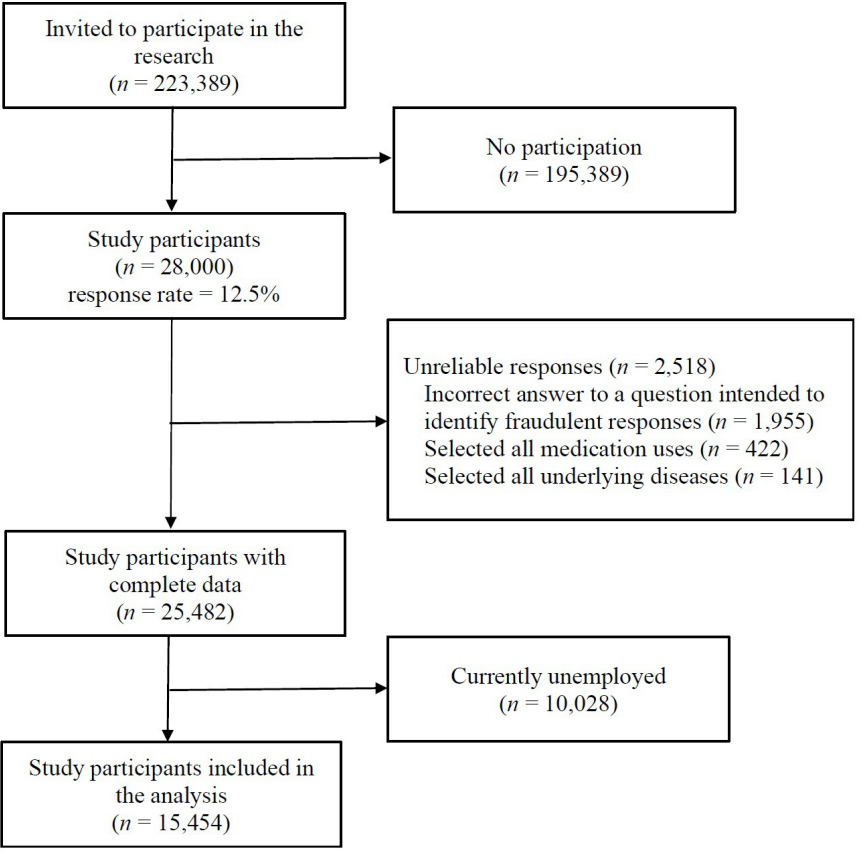

Figure 1 Flow chart of the study participants.

when the target numbers of participants for each sex, age and prefecture category were reached. A total of 28000 participants responded to the survey (response rate $=12.5 \%$ ). We excluded 10028 respondents who reported that they were currently unemployed and 2518 respondents who gave unreliable responses, leaving 15454 respondents who were eligible for analysis (figure 1).

\section{Outcome}

The main study outcome was productivity loss attributed to presenteeism, measured using the Work Functioning Impairment Scale (WFun).$^{20}$ The WFun, which evaluates 'the degree to which the ability to function at work is impaired by health problems, ${ }^{, 20}$ was originally developed in Japan and has shown good correlation with measures of different types of presenteeism that have been proposed by scholars in recent years. ${ }^{20-22}$ The WFun includes linear rating scales on the Rasch model ${ }^{23}$ and has appropriate measurement properties according to the guideline of consensus-based standards for the selection of health measurement instruments. ${ }^{24}$ The WFun contains seven items. Each question asks about the respondent's experience in the last 30 days, meaning that we measured work productivity during August and September 2020 (3 months after the COVID-19 state of emergency was eased). For each question, respondents select from five options scored from 1 to 5 points. Total WFun scores therefore range from 7 to 35 points. Higher scores indicate lower performance at work.

\section{Independent variables}

In this study, we hypothesised that disrupted care has a negative impact on workers' health status and presenteeism, resulting in productivity loss. Here, the scope of care includes all physical and mental illnesses except for 
COVID-19 infection. We asked about three types of events related to disrupted care during the COVID-19 state of emergency (April and May 2020) and one event related to the negative impact on self-reported health status. The three types of events related to disrupted care were as follows:

1. Disruptions in non-routine clinical settings (mostly not medical emergencies but minor illnesses, such as slight fever, wounds, or diarrhoea);

2. Running out of drugs; and

3. Disruptions in routine clinical settings.

The items related to these three event types were 'I could not see a doctor for unscheduled visits,' 'I ran out of routine drugs', and 'I could not see a doctor for scheduled visits.' For each item, the response options were yes, no, and not applicable, which we translated as 'illness with event,' 'illness without event,' and 'no illness.'

In addition, the negative impact on health status was assessed by the exacerbation of underlying disease using the following question: 'My underlying disease got worse,' again with the response options of yes, no and not applicable.

\section{Adjusted variables}

We collected demographic information about gender, age, annual household income, employment pattern, job type and underlying disease from questionnaire data. Employment pattern was categorised as permanent employee, company executive, temporary employee, part-time employee or self-employed. New employment patterns are emerging as the labour market changes. Therefore, we asked about both classic employee patterns (eg, permanent employment) and new employment patterns (eg, temporary employment and self-employment). Job type was categorised as blue-collar, white-collar or other jobs. Other jobs mainly comprised 'pink-collar' jobs such as customer service, retail and nursing care work. ${ }^{25} \mathrm{We}$ also asked about 16 types of illnesses, including hypertension, diabetes and asthma (listed in full in table 1).

\section{Statistical analysis}

WFun score was classified into two groups, in line with a previous study ${ }^{26}: 7$ to 20 points was considered low productivity at work, and 21 to 35 points was considered high productivity at work. A WFun score of 21 or higher requires consideration of accommodations and adjustments in the workplace for workers' illnesses, ${ }^{26}$ and a score of 25 or higher increases the risk of workers taking sick leave. ${ }^{27}$

Univariate and multiple logistic regression analyses were used to investigate the associations among variables related to health status, those related to disrupted care and WFun score. The same statistical techniques were used to evaluate the association between disrupted care and health status. Participants who chose the 'no illness' option were excluded from part of the later analysis. Both analyses were adjusted for demographic factors (gender, age, household income, employment pattern, job type and underlying disease). Goodness of fit was assessed using the Hosmer-Lemeshow test. All $\mathrm{p}$ values were two
Table 1 Demographic characteristics of the participants

n (\%)

\begin{tabular}{|c|c|}
\hline \multicolumn{2}{|l|}{ Gender } \\
\hline Women & $6446(41.7)$ \\
\hline Men & $9008(58.3)$ \\
\hline \multicolumn{2}{|l|}{ Age (years) } \\
\hline $15-29$ & $2326(15.1)$ \\
\hline $30-39$ & $3024(19.6)$ \\
\hline $40-49$ & $4021(26.0)$ \\
\hline $50-59$ & $3301(21.4)$ \\
\hline $60-79$ & $2782(18.0)$ \\
\hline \multicolumn{2}{|l|}{ Annual household income (yen) } \\
\hline Less than 4000000 & $3817(24.7)$ \\
\hline $4000000-599999999$ & $3279(21.2)$ \\
\hline $6000000-899999999$ & $2349(15.2)$ \\
\hline 8000000 and higher & $3522(22.8)$ \\
\hline Unknown & $2487(16.1)$ \\
\hline \multicolumn{2}{|l|}{ Employment pattern } \\
\hline Permanent employee & $8666(56.0)$ \\
\hline Company executive & $847(5.5)$ \\
\hline Temporary employee & $1338(8.7)$ \\
\hline Part-time employee & $2870(18.6)$ \\
\hline Self-employed & $1733(11.2)$ \\
\hline \multicolumn{2}{|l|}{ Labour type } \\
\hline Blue-collar job & $4163(26.9)$ \\
\hline White-collar job & $7498(48.6)$ \\
\hline Other & $3793(24.5)$ \\
\hline \multicolumn{2}{|l|}{ Underlying disease } \\
\hline Hypertension & $2369(15.3)$ \\
\hline Diabetes & $848(5.5)$ \\
\hline Asthma & 549 (3.6) \\
\hline Bronchitis & $220(1.4)$ \\
\hline Atopic dermatitis & $797(5.2)$ \\
\hline Periodontal disease & $1837(11.9)$ \\
\hline Caries & $1688(10.9)$ \\
\hline Ear disease & $173(1.1)$ \\
\hline Angina & $212(1.4)$ \\
\hline Myocardial infarction & $156(1.0)$ \\
\hline Stroke & $131(0.8)$ \\
\hline Chronic obstructive pulmonary disease & $128(0.8)$ \\
\hline Cancer & $238(1.5)$ \\
\hline Chronic pain & $1557(10.1)$ \\
\hline Depression & $583(3.8)$ \\
\hline Other mental health problem & $543(3.5)$ \\
\hline
\end{tabular}

sided, and $\mathrm{p}<0.05$ was considered statistically significant. All analyses were conducted using Stata/SE V.16.1 (StataCorp, College Station, TX, USA). 
Table 2 Exacerbation of underlying disease and disrupted care for employees during the COVID-19 state of emergency by WFun score

\begin{tabular}{|c|c|c|c|c|c|c|}
\hline & \multirow{2}{*}{\multicolumn{2}{|c|}{ Total }} & \multicolumn{4}{|l|}{ WFun score } \\
\hline & & & \multicolumn{2}{|c|}{$\begin{array}{l}\text { 7-20 points (low work } \\
\text { productivity) }\end{array}$} & \multicolumn{2}{|c|}{$\begin{array}{l}\text { 21-35 points (high work } \\
\text { productivity) }\end{array}$} \\
\hline \multicolumn{7}{|c|}{ My underlying disease got worse (exacerbation of underlying disease) } \\
\hline N/A (no illness) & 10917 & 70.6 & 9002 & 72.3 & 1915 & 63.8 \\
\hline \multicolumn{7}{|c|}{ I could not see a doctor for unscheduled visits (disruptions in non-routine clinical settings) } \\
\hline N/A (no illness) & 11496 & 74.4 & 9478 & 76.1 & 2018 & 67.2 \\
\hline No (illness without event) & 3235 & 20.9 & 2511 & 20.2 & 724 & 24.1 \\
\hline Yes (illness with event) & 723 & 4.7 & 464 & 3.7 & 259 & 8.6 \\
\hline Yes (illness with event) & 560 & 3.6 & 334 & 2.7 & 226 & 7.5 \\
\hline \multicolumn{7}{|c|}{ I could not see a doctor for scheduled visits (disruptions in routine clinical settings) } \\
\hline N/A (no illness) & 10103 & 65.4 & 8317 & -66.8 & 1786 & 59.5 \\
\hline No (illness without event) & 3651 & 23.6 & 2881 & -23.1 & 770 & 25.7 \\
\hline Yes (illness with event) & 1700 & 11.0 & 1255 & -10.1 & 445 & 14.8 \\
\hline
\end{tabular}

N/A, not applicable; WFun, Work Functioning Impairment Scale.

\section{Patient and public involvement}

The participants and public were not involved in the design, conduct, reporting, or dissemination plans of our research.

\section{RESULTS}

Table 1 shows the demographic characteristics of the participants. Of 14545 participants, the majority were men (58.3\%), permanent employees $(56.0 \%)$ and blue-collar job $(48.6 \%)$. The most frequent underlying disease was hypertension $(15.3 \%)$, followed by periodontal disease $(11.9 \%)$, caries $(10.9 \%)$, and chronic pain $(10.1 \%)$.

Table 2 shows the number and proportion of each variable related to disrupted care and health status by WFun score. One-fifth of the participants (19.4\%) scored 21 to 35 points on the WFun scale, indicating relatively low productivity at work. In total, 431 participants $(2.8 \%)$ reported that their underlying disease worsened, 723 $(4.7 \%)$ reported that they could not see a doctor for unexpected symptoms or illnesses, and $560(3.6 \%)$ ran out of routine drugs during the state of emergency. A total of 3651 participants $(23.6 \%)$ continued to see doctors as scheduled, but $1700(11.0 \%)$ reported a disruption of their care.

Table 3 shows the associations among health status, disrupted care and WFun score. In the univariate and multivariate analyses, participants who experienced exacerbation of underlying disease or any aspect of disrupted care were more likely than others to show lower productivity at work: exacerbation of underlying disease (adjusted OR (aOR) 2.84; 95\% CI 2.28 to 3.53), disruptions in nonroutine clinical settings (aOR 2.34; 95\% CI 1.97 to 2.79), running out of drugs (aOR 2.58; 95\% CI 2.13 to 3.12) and disruptions in routine clinical settings (aOR 1.67; $95 \%$ CI 1.47 to 1.91). Productivity loss was also somewhat associated with having a chronic disease, even when care was not disrupted (illness without event vs no illness, all $p$ values $<0.001)$. The Hosmer-Lemeshow test confirmed the goodness of fit of the adjusted model $(p>0.20)$.

Table 4 shows the association between the disrupted care variables and health status for those who had any underlying disease. Each aspect of disrupted care was associated with an increased likelihood of exacerbation of underlying disease in both the univariate model and the adjusted model: disruptions in non-routine clinical settings (aOR 6.29; 95\% CI 4.74 to 8.34), running out of drugs (aOR 6.13; 95\% CI 4.60 to 8.18 ) and disruptions in routine clinical settings (aOR 4.64; 95\% CI 3.64 to 5.92).

\section{DISCUSSION}

This study evaluated the association between disrupted care during the COVID-19 state of emergency and health status, as well as the productivity loss attributed to presenteeism. As far as we could establish, our study is among the first to provide evidence about an occupational health problem emerging from the COVID-19 pandemic. Among 
Table 3 Associations among variables related to disrupted care, variables related to health status and WFun score

\begin{tabular}{|c|c|c|c|c|}
\hline WFun $\geq 21$ points & Univariate & & Adjusted* & \\
\hline$\%$ & (95\% Cl) & $P$ value & (95\% Cl) & $P$ value \\
\hline
\end{tabular}

My underlying disease got worse (exacerbation of underlying disease)

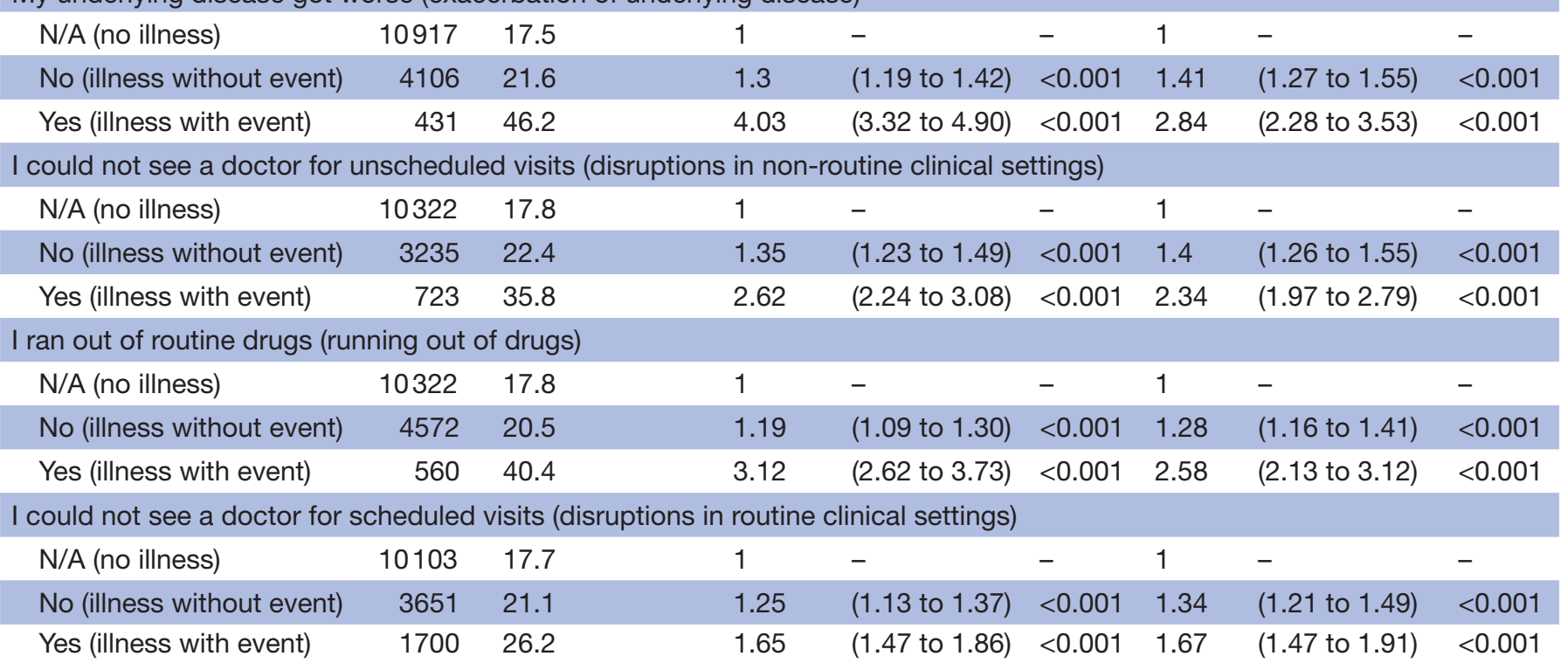

*Adjusted for gender, age, household income, employment pattern, labour type and underlying disease.

N/A, not applicable; WFun, Work Functioning Impairment Scale.

workers, we found that experiencing any of the measured aspects of disrupted care was strongly associated with exacerbation of underlying disease, and workers experiencing disrupted care were also much more likely to show productivity loss. This suggests that reduced accessibility of care for non-COVID-19 patients during the COVID-19 pandemic may have contributed to work performance.

We considered exacerbation of underlying disease as one pathway through which disrupted care could affect productivity loss. The current study found that experiencing disruptions in routine and non-routine clinical settings and running out of drugs were strongly associated with exacerbation of underlying disease. Furthermore, workers experiencing the exacerbation of underlying disease were much more likely than workers without this experience to show reduced productivity at work. This finding is consistent with a previous study: Gerich showed that presenteeism is strongly influenced by the frequency of health events. ${ }^{28}$ Our study provides insight into the possible harmful impact of reduced accessibility of care on productivity loss for non-COVID-19 patients. A global survey reported that $24 \%$ of healthcare providers

Table 4 Association between disrupted care and exacerbation of underlying disease among those with any underlying illness

\section{Exacerbation of} underlying disease Univariate Adjusted*

$\begin{array}{llllllllll} & \% & \text { OR } & (95 \% \mathrm{Cl}) & \text { P value } & & \text { OR } & (95 \% \mathrm{Cl}) & \text { P value }\end{array}$

\begin{tabular}{|c|c|c|c|c|c|c|c|c|}
\hline No (illness without event) & 2950 & 5.9 & 1 & - & - & 1 & - & - \\
\hline Yes (illness with event) & 415 & 32.8 & 7.78 & (6.02 to 10.0$)$ & $<0.001$ & 6.29 & (4.74 to 8.34$)$ & $<0.001$ \\
\hline No (illness without event) & 3156 & 4.7 & 1 & - & - & 1 & - & - \\
\hline Yes (illness with event) & 986 & 20.9 & 7.82 & (6.04 to 10.1$)$ & $<0.001$ & 6.13 & (4.60 to 8.18$)$ & $<0.001$ \\
\hline No (illness without event) & 3855 & 5.3 & 1 & - & - & 1 & - & - \\
\hline Yes (illness with event) & 390 & 30.5 & 5.33 & (4.26 to 6.68$)$ & $<0.001$ & 4.64 & (3.64 to 5.92 ) & $<0.001$ \\
\hline
\end{tabular}

*Adjusted for gender, age, household income, employment pattern, labour type and underlying disease.

N/A, not applicable. 
rated their disease management during the COVID-19 pandemic as poor or very poor, and the mental health of over $80 \%$ of patients worsened during the pandemic. ${ }^{12} \mathrm{~A}$ previous study found significantly higher odds of presenteeism among people with psychological complaints, such as mental health problems ( $\mathrm{aOR} 20.45$ ), malaise (aOR 11.91 ) and sleep problems (aOR 8.62). ${ }^{21}$ Taken together, this evidence suggests that interventions to address health complaints resulting from poor disease management and mental health problems during the pandemic may be important in preventing presenteeism.

The present study found that care in non-routine clinical settings was associated with productivity loss, especially when this care had been disrupted. This finding is consistent with a previous study of emergency department visits during the pandemic, which found that medical care delays and avoidance increased the death toll for people with non-COVID-19 acute illnesses. ${ }^{16}$ A previous study conducted in the US reported that approximately $12 \%$ of adults avoided or delayed seeking emergency care during the pandemic. ${ }^{11}$ In our study, respondents who had experienced disruptions of non-routine hospital visits for minor illnesses (aOR 2.34) were more likely than those who were still able to see a doctor to report reduced productivity at work (aOR 1.40). One possible reason for this finding is that self-diagnosis and self-triage tend to be associated with incorrect diagnoses and inappropriate treatment. ${ }^{29}$ These findings suggest that it is important to receive timely non-routine care during a pandemic, regardless of the possibility of infection.

Running out of drugs and disruptions in routine clinical settings were also related to performance at work. The finding about medication is consistent with a previous study of depression, which showed that depressed employees often experience long-term loss of work performance when they run out of drugs. ${ }^{30}$ Our study also demonstrated that disruptions in routine clinical settings showed a relatively weak association with reduced productivity at work (aOR 1.67) when compared with running out of drugs (aOR 2.58) or disruptions in non-routine clinical settings (aOR 2.34). Family doctors tried to offer patients routine care visits during the COVID-19 pandemic whenever they showed symptoms of exacerbated clinical conditions. ${ }^{12}$ Postponement by family doctors therefore probably did not have much influence on presenteeism. These findings suggest that support for continued medication and timely non-routine hospital visits, rather than routine hospital visits, would help workers to maintain their productivity at work. Such support might include telemedicine and drug delivery services. ${ }^{31}$

This study has some limitations. Because of the study's cross-sectional design, causal relations among disrupted care, health status and productivity loss could not be established. To cope with this limitation, we asked about each issue using different time periods: experience of disrupted care during the COVID-19 state of emergency (April and May 2020) and work performance 3 months later (August and September 2020). However, recall bias may have been an issue. Furthermore, workers who experienced disrupted care might have been more likely to remember health problems, which may have caused an overestimation of productivity loss. In addition, cases of resumed care during this 3-month period may have attenuated the effects of disrupted care. Another limitation is that we did not specify which underlying disease was associated with disrupted care. We also did not evaluate whether underlying diseases developed before or after the outbreak of the COVID-19 pandemic. We adjusted for potential causes in the statistical analysis, but future studies should consider this issue to clarify the relationship between underlying diseases and disrupted care. In addition, the response rate of this study was relatively low $(12.5 \%)$. The results should also be interpreted carefully because of the healthy worker effect. Despite these limitations, this study revealed an occupational health problem emerging during the COVID-19 pandemic and drew on a large sample of nationwide data.

In conclusion, our study showed that workers who experienced disrupted care were much more likely than others to show productivity loss. Exacerbation of underlying disease is one possible pathway through which disrupted care could affect productivity loss attributed to presenteeism. Our study provides evidence of the importance of early diagnosis and continuous treatment of non-COVID-19 patients to enable them to remain healthy and continue to work during the pandemic. Increasing accessibility of care for patients, for example by offering telemedicine appointments and drug delivery, could help workers to maintain their performance at work.

Contributors TT was the chairperson of the study group. TI conceived the research questions. TT designed the research protocol and collected the data. TI conducted the statistical analysis and drafted the initial manuscript with YF. KT, AH, MO and YY revised the manuscript. All the authors read and approved the final manuscript.

Funding This study was funded by Japan Society for the Promotion of Science (JSPS) KAKENHI Grants (grant numbers 17H03589, 19K10671, 19K10446, 18H03107, 18H03062 and 18K10079); a JSPS Grant-in-Aid for Young Scientists (grant number 19K19439); the Research Support Program to Apply the Wisdom of the University to tackle COVID-19 Related Emergency Problems; University of Tsukuba; and Health Labour Sciences Research Grant (grant numbers 19FA1005 and 19FG2001).

Competing interests YF has received research grants and/or personal fees from NTT DATA MSE Corp.; The LOFT Co., Ltd.; Sompo Health Support Inc.; Asahi Shimbun Co.; Chugai Pharmaceutical Co., Ltd.; Asahi Kasei Pharma Co.; AstraZeneca K.K.; Pfizer Japan Inc.; Saibugas Co., Ltd.; Nippon Steel Co.; Hitachi Systems Ltd.; Mitsubishi Research Institute Inc.; and Institute for Building Environment and Energy Conservation.

Patient and public involvement Patients and/or the public were not involved in the design, or conduct, or reporting, or dissemination plans of this research.

\section{Patient consent for publication Obtained.}

Ethics approval This study involves human participants and was approved by the Institutional Review Board of the Osaka International Cancer Institute (No. 20084) and the Ethics Committee of the University of Occupational and Environmental Health, Japan (R3-055). Participants gave informed consent to participate in the study before taking part.

Provenance and peer review Not commissioned; externally peer reviewed. Data availability statement No data are available.

Open access This is an open access article distributed in accordance with the Creative Commons Attribution Non Commercial (CC BY-NC 4.0) license, which 
permits others to distribute, remix, adapt, build upon this work non-commercially, and license their derivative works on different terms, provided the original work is properly cited, appropriate credit is given, any changes made indicated, and the use is non-commercial. See: http://creativecommons.org/licenses/by-nc/4.0/.

\section{ORCID iDs}

Tomohiro Ishimaru http://orcid.org/0000-0002-9334-1423

Ai Hori http://orcid.org/0000-0002-0191-5996

Yoshihisa Fujino http://orcid.org/0000-0002-9126-206X

Takahiro Tabuchi http://orcid.org/0000-0002-1050-3125

\section{REFERENCES}

1 Lerner DJ, Amick BC, Malspeis S, et al. A national survey of healthrelated work limitations among employed persons in the United States. Disabil Rehabil 2000;22:225-32.

2 Skagen K, Collins AM. The consequences of sickness presenteeism on health and wellbeing over time: a systematic review. Soc Sci Med 2016;161:169-77.

3 Johns G. Presenteeism in the workplace: a review and research agenda. J Organ Behav 2010;31:519-42.

4 Schultz AB, Edington DW. Employee health and presenteeism: a systematic review. J Occup Rehabil 2007;17:547-79.

5 Burton WN, Pransky G, Conti DJ, et al. The association of medical conditions and presenteeism. J Occup Environ Med 2004;46:S38-45.

6 Miraglia M, Johns G. Going to work ill: a meta-analysis of the correlates of presenteeism and a dual-path model. J Occup Health Psychol 2016;21:261-83.

7 Kinman G, Grant C. Presenteeism during the COVID-19 pandemic: risks and solutions. Occupational medicine 2020:kqaa193.

8 Lupușoru I, Ciobanu D, Ursaru M, et al. Difficulties in treating a patient with multiple cancers in the COVID-19 pandemic. Chirurgia 2020;115:670-6.

9 Nikolayevskyy V, Holicka Y, van Soolingen D. Impact of COVID-19 pandemic on tuberculosis laboratory services in Europe. Eur Respir J 2020.

10 Erol MK, Kayıkçıoğlu M, Kılıçkap M, et al. Treatment delays and in-hospital outcomes in acute myocardial infarction during the COVID-19 pandemic: a nationwide study. Anatol J Cardiol 2020;24:334-42.

11 Czeisler Mark É, Marynak K, Clarke KEN, et al. Delay or avoidance of medical care because of COVID-19-related concerns - United States, June 2020. MMWR Morb Mortal Wkly Rep 2020;69:1250-7.

12 Chudasama YV, Gillies CL, Zaccardi F, et al. Impact of COVID-19 on routine care for chronic diseases: a global survey of views from healthcare professionals. Diabetes Metab Syndr 2020;14:965-7.

13 Shimizu K, Negita M. Lessons learned from Japan's response to the first wave of COVID-19: a content analysis. Health Care 2020;8 doi:10.3390/healthcare8040426

14 Yabe T, Tsubouchi K, Fujiwara N, et al. Non-compulsory measures sufficiently reduced human mobility in Tokyo during the COVID-19 epidemic. Sci Rep 2020;10:18053.
15 LoPresti M, Seo T, Sato N. PNS263 pandemics and access to care: use of real-world data to examine the impact of COVID-19 on pharmacy visits in Japan. Value in Health 2020;23:S685.

16 Lange SJ, Ritchey MD, Goodman AB, et al. Potential indirect effects of the COVID-19 pandemic on use of emergency departments for acute life-threatening conditions - United States, January-May 2020. MMWR Morb Mortal Wkly Rep 2020;69:795-800.

17 Reuter M, Dragano N, Wahrendorf M. Working while sick in context of regional unemployment: a Europe-wide cross-sectional study. J Epidemiol Community Health 2020 doi:10.1136/jech-2020214888

18 Makishima M, Fujino Y, Kubo T, et al. Validity and responsiveness of the work functioning impairment scale (WFun) in workers with pain due to musculoskeletal disorders. J Occup Health 2018;60:156-62.

19 Ministry of Health, Labour and Welfare. Comprehensive survey of living conditions. Tokyo: Health and Welfare Statistics Association, 2019.

20 Fujino Y, Uehara M, Izumi H, et al. Development and validity of a work functioning impairment scale based on the Rasch model among Japanese workers. J Occup Health 2015;57:521-31.

21 Ishimaru T, Mine Y, Fujino Y. Two definitions of presenteeism: sickness presenteeism and impaired work function. Occup Med 2020;70:95-100.

22 Ishimaru T, Fujino Y, Anzai T, et al. Validity and responsiveness of the work functioning impairment scale (WFun) in rheumatoid arthritis patients: a multicenter prospective study. Mod Rheumatol 2020;30:821-7.

23 Bond T, Yan Z, Heene M. Applying the Rasch model: fundamental measurement in the human sciences. New York: Routledge, 2020.

24 Mokkink LB, de Vet HCW, Prinsen CAC, et al. COSMIN risk of bias checklist for systematic reviews of patient-reported outcome measures. Qual Life Res 2018;27:1171-9.

25 Basu S, Ratcliffe G, Green M. Health and pink-collar work. Occup Med 2015;65:529-34.

26 Nagata T, Fujino Y, Saito K, et al. Diagnostic accuracy of the work functioning impairment scale (WFun): a method to detect workers who have health problems affecting their work and to evaluate fitness for work. J Occup Environ Med 2017;59:557-62.

27 Fujino Y, Shazuki S, Izumi H, et al. Prospective cohort study of work functioning impairment and subsequent absenteeism among Japanese workers. J Occup Environ Med 2016;58:e264-7.

28 Gerich J. Sick at work: methodological problems with research on workplace presenteeism. Health Servic Outcome Res Methodol 2015;15:37-53.

29 Semigran HL, Linder JA, Gidengil C, et al. Evaluation of symptom checkers for self diagnosis and triage: audit study. BMJ 2015;351:h3480.

30 Nagata T, Fujino Y, Ohtani M, et al. Work functioning impairment in the course of pharmacotherapy treatment for depression. Sci Rep 2020;10:15712.

31 Das AK, Mishra DK, Gopalan SS. Reduced access to care among older American adults during CoVID-19 pandemic: results from a prospective cohort study. medRxiv 2020. 